\title{
An Orderliness for Curtailing Human Causalities Shouldered on Networks Associated with Vehicles to Conceive a Blueprint for an ITS for Utilization in Borough Habitats
}

\author{
D. N. S. Ravi Kumar* and S. Barani \\ School of Electrical \& Electronics Engineering, Sathyabama Institute of Science \& Technology, Chennai India.
}

Received 6 May 2020; Accepted 20 July 2020

\begin{abstract}
With the steady rise in the rate of population explosion as well as the growth of the concrete jungles, has led to the need of luxury as a necessity for momentum from one source to a destination in the perspective of an individual. Today's Technology has overridden the functioning of Humans, so much that the mechanical nuts and bolts has become a part and parcel of an individual's life. The above thought flow has conceived the sale of ' $n$ ' number of four wheelers to move about in Cities and Townships. The aftermath of the above entities have set out a chain of debacles for the concerned jury with respect to the orderliness of traffic arising on account of Four wheelers. The repurcussion of the aforementioned comments have paved a path for a bottleneck associated with gridlock, exponential upsurge in civilian casualties, and last but not the least the discharge of impurities let out from the exhaust hose of the Vehicles. The casualties which are being experienced by Individuals turn out to be the crucial motive behind the loss of livelihood, regardless of the evolution of cosmopolitan orderliness associated with gridlock governance, coupled with diverse automations associated with four wheelers. Therefore, at this juncture the conceivement of a centralized orderliness associated with gridlock governance related to vehicles becomes a dire necessity. For citing an event in a real time scenario, the grid lock caused by vehicles in utmost townships can get assuaged by means of devising a frame work that is efficient in working in real time, for the routes that is supposed to be taken by the four wheelers. Nonetheless, tailoring a contrivance for planning a route for four wheelers with a touch of efficiency in nature, as well as for conceiving an orderliness with an attribute of universal, flawless dominion over the vehicles remain a debacle that needs to be answered properly, specifically when the consideration leans towards the preferences which are exclusive related to the individuals behind the wheels. The desideratum of this manuscript focuses on the conceivement of an orderliness that has governance, over the incidence leading to human casualties or mishaps to curtail them from happening in the future, and the above mentioned entity utilizes Networks without the presence of a Router, merged with gadgets that engages the technology of Cellulars, pasted in transportations availed by the public. The aforementioned orderliness or system possessing the caliber for communication, assures a secure hand shake between Four Wheelers, Vehicles handling the Patients, Healthcare Units, Road side Assistance, as well as the backbone of the show, which is nothing but the Centralized Servers. With an addition to the above statements, the orderliness that has governance, over the incidence leading to human casualties or mishaps, has the ability to broaden the golden hour, by igniting a red alert to fast forward an Ambulance to the scene of a human casualty, by utilizing an algorithm that teleports the red alert from the scene of human casualties to the Healthcare Unit, via Multiple Hops. Above all, a set of codes to perform a possible planning of routes, that is supposed to be followed by four wheelers gets provisioned here, to trump up the hotchpotch dimensional utilization of networks related to road, while cutting down the expense of operation over a specific vehicle. The required trial and error method is done for the purpose of studying the set of codes, and the outcome gets correlated on par with the pre-existing set of codes. The outcome of the defined trial and error methodologies cater a deposition, that the provisioned set of codes far exceeded the expectations and has taken the limelight in terms of attributes like the rate of acceleration of the Ambulance, as well as the requirement of time for reaching the spot where the casualty has occurred. Last but not the least, the orderliness which is getting focused in the manuscript, charts out a beautiful path for an Ambulance to breeze through roads which seemed to be glued with vehicles, therefore the survival rate of Individuals gets broadened from 50 to 95 percentile.
\end{abstract}

Keywords: ITS, Casualty Management, Authentication

\section{Introduction}

The notion of conceiving a city or a corporation to function smart is closely associated to a fundamental fluctuation wherein the passion is focused upon the overtures as well as the drive related to technology, that endeavors the transformation of the citizens with an aura of positive effect

*E-mail address: rk9980@gmail.com

ISSN: 1791-2377 @ 2020 School of Science, IHU. All rights reserved.

doi:10.25103/iestr.134.06 on the standard of livelihood, as given out by the authors in [1] and [2]. To retrace a page from the history associated to cities becoming smart, we have the commission which got incubated in Europe went on to become the trend setter in conceiving the ever first Initiative which was smudged on the ideals of converting the normal towns and cities to smart entities. The above mentioned initiative focuses on handling four trivial traits with absolute discretion, they are as follows:

An orderliness that has an authority over the Governance of the Weather, Tesla's Flow of Electrons, Concrete 
Structures, and last but not the least Man's invention that started the entire show, the means of traversing from one source to another destination: The Wheel. One of the primary entities that the cities which deem to be smart, has taken up an assignment which is focused on the arrest of mishaps that exponentially has a toll on the human casualties. The increase in the production and the utilization of four wheelers have a two hundred percent impact on the life span of Individuals, as well as the formation of a tight grid lock on the pathway utilized for exodus. The Writer in Manuscript [3] has stated that there occurs a toll on the life of more than one hundred individuals day in and day out on account of the mishaps arising from improper handling of four wheelers, where in the person stepping on the gas, being in an inebriated state. The survey with reference to the manuscript[3] focuses a boom light upon the year $2 \mathrm{k} 7$, which accounts for more than half of a lakh human causalities which has a direct proportion to the number of mishaps occurring due to the worst handling of four wheelers in terms of statistics ranging from five to ten million in mathematical numerals. Furthermore, among the majority of the terrains that occupy some land masses in this planet, it is noted that the dominant motive behind the loss of human lives is the absence of a blueprint which is vital for governance over the gridlock caused by the four wheelers. We can express our point of view and state that the brainchild behind an orderliness which is responsible for governing the gridlock caused by the four wheelers pin points to an astute contrivance utilized in ordinary civilians transportation, which is completely rooted on digital $0 \mathrm{~s}$ and $1 \mathrm{~s}$, being generated on the fly. The above mentioned entity aids the individual behind the steering wheel, to avert directions that might be clogged with ' $n$ ' number of four wheelers, as well as sharpening the aspect of inviolability resulting in the four wheelers to engage in a tone, which is quite amicable to the neighborhood in which individuals reside in. With a span of time ranging from the yester years to the present day, the academicians as well as the scholars prevailing from the two corner stones of a citizen's livelihood i.e. Enterprise as well as Institutions, which are nothing but the two sides of the same coin, have exploited the caliber of WSNs in a way which is quite useful for mankind in ' $n$ ' number of ways. With reference to the above quote, the research giants have marched together to enhance the pre-existing orderliness which governs over the gridlock arising due to the four wheelers, to make it exponentially competent as well as hone its caliber to take care of the back locks arising in a city deemed smart, with the reference to the above. A red flag has been raised in terms of the grid lock which has risen due to the rise in the number of four wheelers, being produced day in and day out from the four wheeler production department. The above has been mentioned as a virus which is slowly munching in on the livelihood of civilians in this modern era. The tardiness which exists in the appearance of the most on demand and the need of the hour trivial entities, like Cops, Emergency Response Team, Grey Hounds, Battalion, Officers of the Fire Search and Rescue Crew as well as the proper functioning of the above mentioned Team of experts at the place of human casualties, Riots or Crowd Control happens on account of the worst grid lock which take place on account of four wheelers. The above mentioned circumstances appear to be a threat to the livelihood of commoners and civilians; each and every moment counts when we discuss about the utmost well being as well as the security of the Individuals, why because the life of an Individual caught up in a mishap depends on the proper timely arrival of the Vehicles associated with Healthcare as well as
Law and Order in the most effective manner with the least amount of time traversal as possible. The entity defined as

The Orderliness conceived for the purpose of transportation which is deemed to be intelligent, is not in a position to overcome the shortcomings which emerge due to the gridlock which arises due to the exponential increase in the four wheelers. To cite an instance, the Play Store is filled with Apps for guidance, from one source to the next destination. To name a few we have 'here', 'G-Maps', Maps based on Google, which depend on the GPS of an end user's Android, Windows or IOS Phones. The above mentioned entities utilize the caliber of pre-existing technologies like Wireless Fidelity, as well as the pre-defined Positioning Systems coupled with the Networks based out of Cellulars to sketch out the pathway from one source to the required destination based on the vested interest of the end user, coupled with handling the worst setbacks of being glued to one particular spot for lengthy hours due to the grid lock of four wheelers. Even though these pre-existing technologies might seem to be quite expensive, they do not possess the caliber to communicate to and fro in a timely manner, when a circumstance arises on account of mishaps leading to human causalities. The above mentioned technological devices have a short coming in terms of reading and interpreting the data given out by grid locks formed by the four wheelers.

Therefore it is quite trivial that the above mentioned entities ought to get installed with an orderliness that has the ability to increase the adaptation of forging a suitable path way, which in return increases the efficiency to basket the data as well as the digital data emerging from on the fly grid lock may get over used in a positive manner to avert the clogging of four wheelers on the road. Primo, the transpiring entity defined as the Ad hoc Network associated with the Vehicles can get utilized to shower upon the system, rooted on an orderliness associated with Transportation, which possess an improvised caliber for establishing a to and fro communication for acquiring an on the fly data which is associated with the gridlock conceived by the Four Wheelers, provided the acquisition is gained in an efficient and in a manner which is effective in terms of the cost [4]. The Manuscripts conceived by the authors referenced under [5], [6] as well as [7] \& [8] states that the entity defined as the Ad hoc Network associated with the Vehicles have the ability to bolster the exchange of data to and fro, from the units placed on the appropriate positions on the road onwards the four wheelers and other vehicles traversing on the road. Therefore the data getting generated may get sent and received on the fly with respect to the units placed on the side of the roads as well as the vehicles. The data which is getting generated on the fly, getting accumulated under one basket can get utilized for the framing of a pathway in terms of distinct four wheelers [9], governance over the flow of four wheelers [10, 11], as well as for the localization of vehicles [12-14]. In accordance to the writers of Manuscript referenced under [15] as well as [16], the moment once the data which gets generated on the fly, 'n' number of codes associated with Computer Programs may get conceived with an intention of uncovering the best pathway, which is also quite efficient in nature so that the four wheelers may utilize to reach their respective destination. Nonetheless, when the collegiality takes an unwanted diversion in the event of the fabrication of a path way for the four wheelers, it would pose as a bottleneck for free flow of the vehicles. Whilst the majority of the Computer Programs which are handling the fabrication of pathways are comprehensive in nature, are concerned only with the improvisation with anything and everything associated to the 
concept of Networking, and have a negotiable attention to the entities like the interests of the person behind the Wheel, the span between two entities, and last but not the least the measurement of Time. The above discussed statements are extremely crucial, in the event when an individual has a thought flow that the verdict associated with replanning arises due to the aftermath of a requirement to avert the grid lock caused by four wheelers as well as to have a control and to balance the flow of vehicles as inimical pointing to the revelation of routes which are deemed to be optimal in nature.

Therefore, when the perspective of the individual behind the wheel is taken into account, a few of them might be interested in driving for a longer and a smooth path without any pot holes, though the path might have a cost much higher when compared to smaller paths. Thence the codes utilized to conceive a Computer Program ought to occur in a way, which has a focus on deduction of the cost for that path and with the least amount of grid lock from the four wheelers. At this juncture, the most trivial beneficence of this manuscript is enumerated below. 1. An orderliness for governing the casualties from ever happening to Human lives, which utilizes Ad hoc Networks associated with Vehicles gelled with the Computers that engages the technology based out of Cellulars in the transportation domain used by Civilians. The system or an orderliness takes care of on the fly exchange of to and fro data between ' $n$ ' number of four wheelers as well as the Central Node Servers. Source codes which controls the behavior of the Computing Systems get utilized in this particular system or orderliness, in turn provisions the path deemed optimal between two entities, aka the four wheeler caught in the human causality as well as the Vehicle associated with the Healthcare Units. The above brings down the golden hour between the causality and the arrival of the Paramedics to the required spot. 2. A path which is optimal for the Paramedics to get to and fro from the Health Care Building to the spot where the mishap had taken place and back again to the Building gets provisioned, by means of a Programming Code which is defined by a generic term called as the Algorithm. The above-mentioned entity engages a communication rooted upon an Ad hoc Network based on Transportation utilized by normal civilians, in order to alleviate the issue of bottleneck created by Four Wheelers. Focusing on the desire of harmonizing the interests of the individuals behind the Wheel, as well as for the purpose of creating a proper flow in the movement of vehicles, two entities are under scrutiny (i) the outlay for the vehicle to commence from source to the destination, (ii) the exertion of the space in a Network. The remnant of this particular manuscript is formulated in the way described as the following:-The category described under the numeral 2 focuses on the entity of Allied Works associated with the planning of a pathway, whilst the category described under the numeral 3 focuses on the Blueprint of the System or an Orderliness. The numeral 4 spotlights the codes utilized for showing the proper pathway for the proper flow of four wheelers, whilst Category 5 pin points a virtual execution associated with the evaluation of performance, and Category 6 puts an end to the Manuscript.

\section{Pertinent Endeavours}

There exists ' $n$ ' number of aftereffects which are associated with dual entities, one being the flow of the four wheelers in an unbalanced manner and two the consequential human casualties coupled with the episodes caused with reference to the above: For an instance an Individual's Tardiness coupled with experiencing an extra fee are a few of the outcomes the person behind the pedal ought to cope up with. The entities associated with Navigation as well as Forging a particular route has the caliber to mollify the shortcomings associated with Gridlock caused by Four Wheelers. The Authors in Manuscript depicted under numerals [17] to [19] expose their mindset by pointing out the fact that governing and forging the proper pathway for four wheelers embeds three variant entities like Navigation of Four Wheelers via Global Positioning System, Reserving a particular pathway before hand, last but not the least the predictive analysis time frame associated with a Human Casualty. Nonetheless, the whole lot of the methodologies mentioned fail to possess the ability to give out on the fly data in the event when a casualty or gridlock takes place in an unexpected manner, acknowledging the factor that the mentioned methods lack the caliber to amend the data associated to the prevailing conditions of the gridlock created by the four wheelers and give it out in an on the fly manner. Therefore, the on the fly data which is associated with gridlock caused by vehicles churns out to be quite trivial when it comes to bolstering the Algorithms which are related to devising the pathways for the four wheelers.

There exist two variant entities, titled Cellular System \& Loop Director that seem quite trivial when it comes to utilizing them in an ITS coupled with basketing data associated with gridlocks that tend to change with respect to time. The Manuscript Developer depicted under [20] and [22] has explained that there was a scrutiny related to the utilization of non-stationary Sensors with Cellular Networks, for the purpose of accumulating on the fly data associated with gridlocks for predicting the flow of traffic. The Author in Manuscript numbered as [15] provisions an orderliness for governing the flow of traffic by utilizing an entity defined as Loop Detector for the purpose of supervising the flow of traffic 24/7 coupled with the observation of trivial pathways. Nonetheless, both the entities have their own positives and negatives. The first entity has its own unique functionality and cannot be forced or influenced to basket the data associated with the flow of traffic and provided sending minuscule amount of data is literally costly. In the event when more than the required amount of data gets shot out via these networks, the data would in turn lead the entire network to crumble like a stack of cards on account of accumulated gridlocks. Deployment of Loop Detectors would also seem to be getting a hit on the cost factor. The Manuscript writer in [23] has given his thought that in the event, there needs a flow of information from the source to the destination for a negotiable distance, the data associated with inappropriate positioning would give rise to many issues, and when the above event happens in a dense network with ' $n$ ' number of Computers, it has a direct effect on the planning of governance of a pathway. The Manuscripts numbered [24] and [25] speak of tri entities named as V2V, Vanet and the last V2R technique of transmitting and receiving data have the caliber to send messages in a quick manner, embedded with attributes like efficiency, cost-effectiveness and much effective on par with the pre-existing methodologies, when pointing out the factor of negotiable distance as well as thick networks. Above all, with reference to VANET the gathering as well as the circulation of data get enhanced by, the units placed on the sides of the Roads, making it feasible for achieving a proper pathway planning for the four wheelers [26]. 'N' number of scrutiny have shed their light upon on the fly planning of a pathway shouldered on VANETs. 
A methodology for circulated planning of pathway, got some advancement to take care of the shortcomings, arising on account of grid locks via the utilization of on the fly 0 s and 1s conceived through a VANET, on account of the escalation in the momentum of vehicles [15]. The Author referencing Manuscript number [29] has conceived a system of navigation that which aids the Individual behind the steering wheel to choose a pathway which is negotiably free from gridlocks, in return has a direct positive effect on the burning of gasoline. Nevertheless, the absence of co-ordination, cooperation coupled with the egotistical conduct of the chauffeurs would in return give rise to traffic bottle necks, in the event when the entity defined as scheme related to user manual gets set up. The Manuscripts numbered under [30] as well as [31] discuss about the planning of pathways for ' $n$ ' number of vehicles. Nonetheless, the works described here turn a deaf ear to the predilection of chauffeurs, or the median cost required for the four wheelers to traverse from one source to the destination. The hypothesis on the reason for which the communication which resides in VANET has a direct negative effect on the Algorithm associated with the planning of pathways for the four wheelers, still remains a mystery. Therefore, in this scrutiny, an Algorithm associated with planning of pathways that which is optimal on a global scale gets provisioned to aid the four wheelers to avert the gridlock caused by the flow of vehicles in order to arrest the human casualties from ever happening in an urbanized environment. A proper expectation has been set up, in such a way that the basketed on the fly data associated with the flow of traffic as well as the decision taken for conceiving a proper pathway, would be of greater help for utilizing the given resources present inside a network related to a pathway, which would have a positive effect in the reduction of the median cost required by a four wheeler to traverse from the source to the destination.

\section{The Prototype and the Blueprint Associated with the Orderliness or System}

Within this given category, the desideratum points to provision an orderliness or a system to arrest the casualties which is happening to the Individuals on account of vehicles with ' $n$ ' number of wheels. In terms of the first order of business, two entities get kicked in, one being the prototype and two the Blueprint associated with the Orderliness or the System under the Study.

a) The Prototype associated with the Orderliness or System: With the reference to the Individuals habituated to a City and a Township, a Network which is shouldered on the Vehicles which utilizes several roundabouts and the respective pathways, gets acknowledged here. Each and every pathway or in accordance to a layman a road conceived by Mr. McAdam, possess dual alleys through which ' $n$ ' number of vehicles traverse in a direction which is contradictive to each other is figured out under the Diagram One. The spherical ellipse, which is present in the Figure holds the position for the ID, which is associated with a roundabout, whilst the traversal of four wheelers; with respect to an alley is denoted by an arrow painted in yellow. The Healthcare Units numbered 1 and 2 are assumed to be the port of call, and are glued between duo cross roads. The units which are present along the sides of the roads get positioned within each and every cross road. Each and every health care unit is equipped with six wheelers, transporting Patients from a source to a destination. Just like the organs of an Individual are interconnected to the Central Nervous System, like wise each and every nuts and bolts of the Orderliness are sinewed to a Centralized Server. A normal four wheeler has a momentum from one point to the next, but over here the points are one and the same for two or more vehicles or they might be different in terms of the traversing direction. The Author in the manuscript depicted under [32] has inscribed a pre-conceived idea that all the Paramedical Team utilizing the vehicles as well as the four wheelers possess a state of the art direction mechanism as well as the caliber to utilize to and fro communication sans the application of 802.3 standard cables. Inbuilt within these Paramedic vehicles and normal four wheelers, there exists a digitized analog chart which charts out the locality of the cross roads. Added to the above, almost ' $n$ ' number of four wheelers posses the efficiency to harness the required data associated with the traversing direction of four wheelers, the locality, the rate of acceleration, and the above gets penned down in the form of a datable which is associated with Tables, columns as well as rows. The above mentioned Data storage get transmitted to and fro via exchange of messages happening from time to time. In the event of generalizing, an assumption gets conceived that ' $n$ ' number of vehicles send out data in a specific frequency, coupled with the pre-conception that the node which is defined to be the source is garrisoned with a mechanism to evaluate the locality of the destination, as when the need arises [33,34].

b) The Blueprint of the Orderliness or System: There exists five arms with respect to the Orderliness which is utilized for governing the casualties, in a way of arresting them from ever happening, and it can be found in the Diagram mentioned under the Numeral 2. The penta entities are as follows: One being the Unit associated with Vehicles, Second being the Centralized Server, Third relates to the Units which are present along the sides of the Macadamized Roads, Fourth the unit which is associated with the Vehicle being utilized by the Paramedical Team, last but not the least the Healthcare Unit. Let us have a crystal clear comprehension of all the above entities.

The Units associated with the Vehicles:-The responsibilities of the Units associated with the Vehicles focuses on the governance of the entire work opted by the four wheelers, which inculcates the induce of red beacon as and when a causality gets triggered, as well as maintaining the life of the packets being exchanged with reference to the other units at hand. Although the Human Body is controlled by the central nervous system, it is delegated for controls of the Vision, Audibility and much more. Likewise there are trio different entities, which are associated with the Units which are relevant to the Vehicles. They are as follows, One being the Segment associated with the Sensor, Two the Segment associated with Monitoring the Rate of Acceleration, Segment related to Messaging and Triggering an Alert.

c) The Segment Associated with the Sensor: The Segment gets utilized for supervising, observing as well as governing the variant segments glued to the four wheelers. The orderliness at hand utilizes dual segments, on account of ascertaining the causalities: the segment associated with, the Science of Biology and Medicine that which ascertains the beat of the Human Heart, the rise in an individual's body heat, as well as the Pressure churning inside an Individual, and the second segment which is associated with the vehicles takes care of observing and reporting, the rate of acceleration, the measure of reverberation, and last but not the least the 
placement of the material to engage and protect the skull of an individual from impact on the steering wheel. The dual Segments have both their positives and negatives. For the purpose of citing an example, the sensor which is associated with the four wheeler is quite facile to utilize, even though it fails to chart out a clear picture on how a particular mishap might have taken place. Whilst the sensor has the caliber to ascertain that a mishap has taken place, the sensor fails in giving out the nitty-gritty data with regard to the importance of the mishap caused by the four wheelers, and what is the depth of the necessity of medical help, that is supposed to be given to the individuals who are caught in the mishap. Nevertheless, the sensor associated with biology and medicine aresome time erroneous in nature, and might trigger a falsified alarm. For the purpose of diminishing the short comings, an idea gets kicked in for utilizing the dual sensors in a parallel way. For anything and everything associated with the sensor, there exists a Uni Processor that handles the digital $0 \mathrm{~s}$ and $1 \mathrm{~s}$, present inside the four wheelers. The Uni Core Processor gets utilized for etching the value which is at its verge, and the value being conceived by the Sensors.

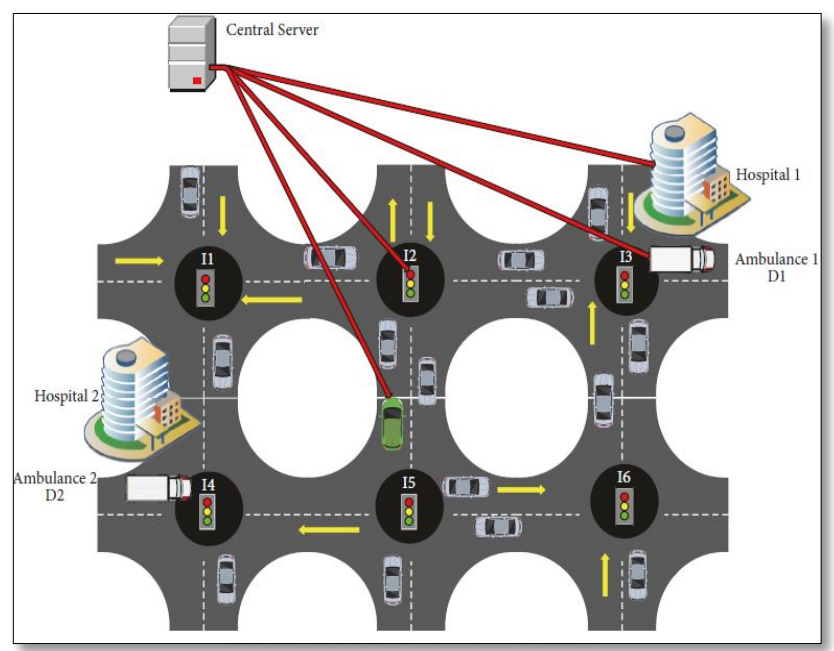

Fig.1. Model of accident management system.

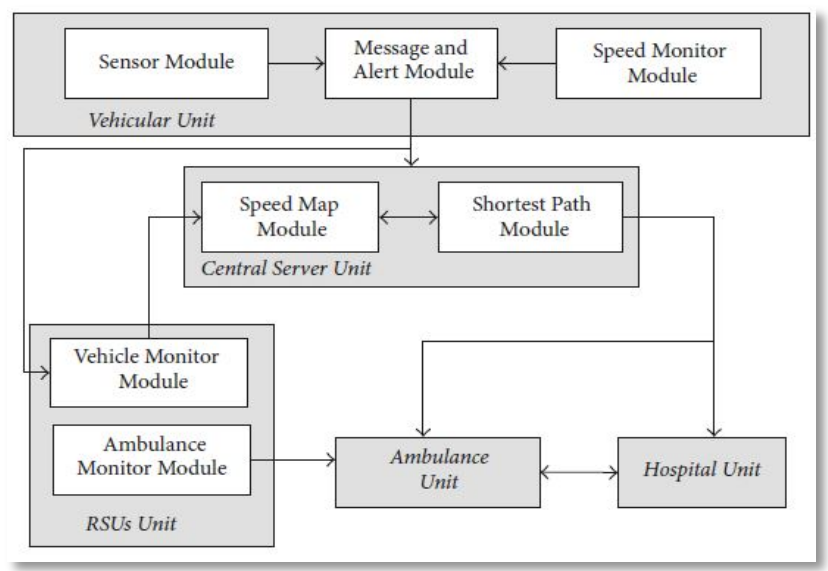

Fig.2.Architecture of accident management system

The responsibilities of the Uni Processor is to gauge and compare the data received on par with the verge value. In the event of any sort of an abnormality, that might arise in the comparison of the received values, the Processor raises a flag for a mishap has certainly taken place. The Data regarding the Human Causality is taken from the Uni Processor and shot to the Segment deemed as Alert. The Uni Processor has a direct access to the data associated with the locality of the Global
Positioning System present inside the Four Wheeler. On account of the above, the data which is associated with the locality of the four wheeler gets tracked in a continuous manner.

The Segment associated with Observing the Rate of Acceleration:-The segment gets utilized to observe and take note of the data associated with the rate of acceleration of a four wheeler, as well as the median acceleration by which a particular four wheeler traverses on each and every pathway. The observed data is passed on to the Segment related to the Messaging and Alert.

The Segment associated with Alert and Messaging:The segment which is relevant to the above gets utilized to govern the exchange of data between the Centralized Servers, the units associated with the sides of the Roads, and last but not the least the Paramedical Team deployed from the Health Care Units. The Segment drags the data related to the median acceleration from the Segment associated with Observing the Rate of Acceleration. This churned in data gets pushed on to the required Unit placed on the sides of the Roads.

The Unit associated with the Centralized Server:-The above mentioned entity forms the centre to the Blueprint, and it has connectivity to ' $n$ ' number of units relevant to the entities. Managing the flow of traffic is the sole role of the Centralized Server. The above mentioned entity comprises dual Segments:

The Segment related to the Mapping of the Acceleration: This Segment utilizes the median acceleration on which the four wheelers are traversing, in order to conceive a digital mapping of the pathways of each and every Roads.

The Segment Associated with the Optimal Path: This segment gets utilized to frame the most comfortable channel, that a Paramedical Vehicle may follow for reaching the scene where the mishap had taken place and back to the Health Care Unit.

The Units present on the side of the Roads:-The roles and the responsibilities of the unit under study speaks of observing as well as governing the flow of traffic, and above all it takes in two variant segments.

The segment associated with observing and studying the Four Wheelers: This segment gets utilized to basket the data of the median rate of acceleration, by which the four wheelers are traversing in a given pathway. The above data which is gained is shot up the Centralized Server.

The Segment associated with the supervision of Paramedical Vehicle:

This Segment gets utilized for geo locating the Paramedic Vehicle as well as configuring the Red, Amber as well as the Green Lights in the pathway, so that the amount of time required for the Paramedical Vehicle may get negated exponentially. The segment under our supervision partitions the Roads into smaller blocks or chunks existing in between dual units present along the side of the Roads.

A unique ID represented by a numeral gets provisioned for each and every block of the Roads. Each and every unit associated with the side of the Road, shoots up this ID, which is going to get used up by the four wheeler which is intending to go through a chunk of the Road. The moment every particular four wheeler has received the data in the form of a message, the rate of acceleration gets recorded on that particular chunk of the Road. The time span by which when a four wheeler reaches the finishing line of a particular chunk, 
a data in the form of a message gets shot to the Units placed along the side of the Road. Present inside the data, are dual entities: one being the Numeral representing the ID and two being the median rate of acceleration. The units present along the sides of the Roads, basket the data related to the rate of acceleration of the four wheeler and utilize this data for evaluating the median acceleration of all the other four wheelers, and this data gets lined up from the units present along the Roads onwards the centralized Server, and on receiving this data, the Server forms a digitized Chart with all the pathways along with the rate of acceleration for all the four wheelers.

The Paramedical Unit: The unit associated with the Vehicles as well as the Centralized Server provision the Paramedical Team with dual data. One being the latitude and longitude of the place where the human causality has taken place coupled with the most favorable path for the paramedical team to pull through the flow of traffic to reach the required destination. With the aid of the Global Positioning System, the Paramedical Team forms the favorable path to the place where the mishap had taken place. Whilst the Para Team is in transit, a small piece of data gets sent out, being identified as the Alert, in turn consisting of dual fields: one being the numeral associated with the ID which is current as well as the next. At the commencement of each and every chunk of the Road, the ID present at that time which is associated with the Road gets pushed on to the unit prevailing at the finish line of the chunk in the Road, by the units in the side of the roads upon getting a triggering data. Post receiving the data, the information which is concerned with the path way taken by the Paramedical Team gets ripped off by the units placed along the sides of the Road, and it is based on this piece of data the lights of the traffic gets altered for the proper vrooming of the Paramedical Vehicle towards the scene of the incident.

The Healthcare Unit:-The tri entities defined as the Health care unit, the Paramedical Unit, and the last but not the least the Centralized Server Unit are woven to each other to form a to and fro communicative paradigm. When the Paramedical Team arrives at an incident, the attributes of the Individual who has suffered the mishap gets recorded as soon as possible inside the Paramedical Vehicle and it further gets transmitted to the Health Care Unit. Therefore an assurance gets established that when an individual reaches the Health Care Unit, the best of the treatment gets done based on the causality endured by the Individual. On account of the fact, that the health care unit keeps a record of the locality of the Paramedical Team, the report of the current situation of the Patient is known by the Health Care concern, several hours even before the Para Medical Team could enter the Health Care Unit. On account of the locality of the Paramedical Team gets updated on to the Health Care Unit, the time frame for when the patient may reach the Health Unit gets evaluated on the fly, therefore the emergency procedures which are required to get carried out is done before hand, so precious minutes is not lost in saving the life of an Individual.

\section{The suggested algorithm associated with routing the flow of traffic}

With reference to this particular chunk, the activities that is supposed to be performed after a human casualty has taken place on account of a vehicle gets discoursed in this orderliness under study. The process of Routing the flow of Traffic is equipped with dual prospective Algorithms. The Primary Algorithm which is responsible for providing a pathway embedded with optimality between a Paramedical Vehicle and a Vehicle which was subjected to Collision is termed as a Forwarding Algorithm based on Multiple Hops embedded with the concept of optimality. The secondary Algorithm which handles the pathway that the Paramedical Vehicle ought to take from the Health Care Unit to the spot where the collision had happened and then back to the Health Care Unit, is christened as the Algorithm planning the Route, embedding Optimality.

a) The Forwarding Algorithm based on Multiple Hops embedded with the concept of Optimality: This block provisions the nitty-gritty information on the ways and means of utilizing the entity referencing the Four Wheeler under a mishap for the purpose of id-ing the pathway to be optimal for reaching the destination six wheeler termed as the Paramedical Vehicle by means of hopping from one point to the next. The terminology hop is extracted from the Networking realm where the packet moves from one Routing device to the next, until the required destination gets reached. The above mentioned algorithm mimics the exchange of information seen under V2V. The Diagram referenced under the numeral 3 exposes the following by which the Vehicle painted in Green is taken as the Source and the Paramedical Vehicle initialized as Numeral 1 is taken as the First Destination which is pin pointed by the Alpha Numeral D1. The Location of D1 is placed under by one more Alpha Numeral I3. The second Paramedical follows the same pattern under the Alpha numerals D2 as well as I4. In the event if it all a collision had taken place by different vehicles, the entity defined as the source shoots out a networking data packet onwards the Paramedical Vehicle which is present in a locality which is closer to it. The vehicle which is under a crash or a collision does a mathematical computation for measuring the distance or the length of the pathway with respect to all the entire junctions that which have a direct link to the Paramedical Vehicles and it appropriately decides the one with the shortest length, denoted by the alphabets PL. With the reference to the alpha numeral D1, focusing the diagram depicted by the numeral 3 , by means of utilizing the red Arrows we find that the calculation referencing PL2D1 is lesser than PL5D1, and above all the packet when shot out from junctions represented by alpha numerals I2 to I3 onwards the alpha numeral D1 is way faster than its counterpart I5 to I6, and I6 to I3. In accordance to the above the human casualties occurring through the collision of vehicles would require dual junctions to reach a Paramedical Vehicle.

1. In the mean time, with a reference to the alpha numeral D2, as depicted in the figure reflected by numeral 3 painted with green arrows, the combination of Path Length and Distance 5 and 2 is less when compared to PL2D2, the distance taken by a network packet to reach the junction I5 to I4 and towards D2 would deem quick and fast than the counterpart I 2 to I1, and I1 to I4. With reference to the above computations the vehicle that met with a collision would opt for the pathway which possess ' $n$ ' number of vehicles for the surety of uninterrupted communication.

Wherefore, dual junctions are mandatory for the vehicle caught up in a mishap, for the sole reason for getting closer to Paramedical Vehicle \#2. The statement above signifies that there exists dual pathways where the trait of distance is somewhat analogous: Junctions I5 to I4 to D2 as well as I2 to 
I3 to D1. With the reference to this instance, it would deem very much appropriate for a vehicle caught up in a pileup to single out a pathway that consists of ' $n$ ' number of four wheelers in such a way that uninterrupted communication gets sustained. With reference to the diagram depicted by numeral 3, the pathway preferred by the vehicle caught in a pileup is denoted by Path Link \#2.

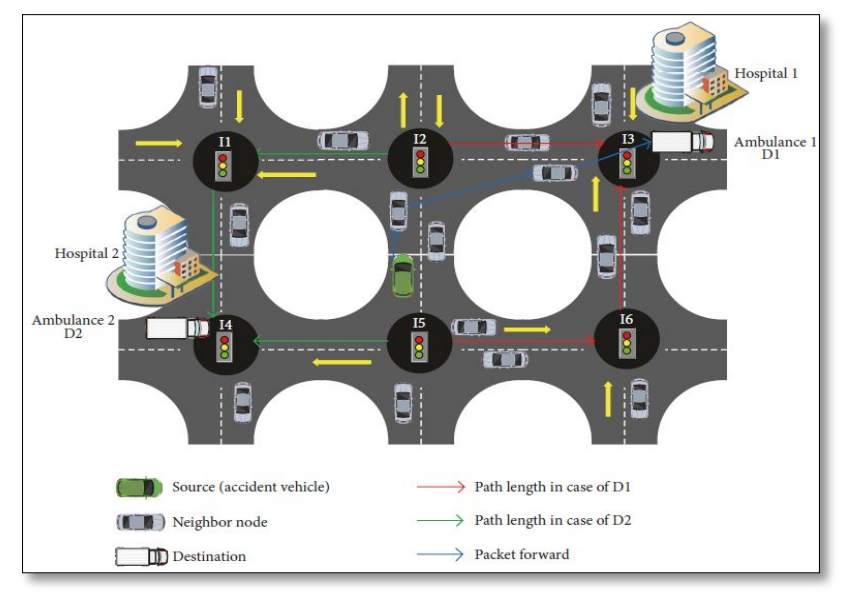

Fig.3. Source vehicle calculates the path length to destination

The Manuscripts denoted by the numerals [35] and [36] expose the fact that the arrows painted blue points the vehicle caught up in a pileup which is pushing the network packet onwards the next consecutive four wheeler which is in momentum towards the junction which possess the diminutive PL towards D1. The above is nothing but a sequence of steps which carries on throughout until the network packet comes at the assessment. The above set of procedure makes sure there is increase in progress, while making $100 \%$ surety, that there exists only a minimized bottle neck created by vehicles on a given Network.

b) The Algorithm planning the Route, embedding Optimality: The desideratum in this block is to single out the Algorithm planning the Route, embedding the concept of Optimality abbreviated as ORPA commencing from the Paramedical Vehicle to the place where the vehicle is being held up in the pileup and then back to the Health Care Unit. The inference which exists behind this particular Algorithm speaks of averting the bottle neck conceived by the vehicles as well as the coupling of the contraction of median traversal cost. It is predicted that each and every four wheeler ought to take a trial ride or a drive a pre-destined pathway from the location where the vehicle embarks its odyssey to the end point leading to the cessation of the traversal. With reference to this particular occurrence the pre-destined pathway gets etched on the Global Positioning System as a default path, which is completely dependent two entities, one being the location of commencement and termination of the traversal, and two points out to the decision of the person behind the steering. The Chauffer is contemplated to obey the pathway chartered out in the GPS unless and until and a Red Flag is hoisted to proclaim a bottleneck has been conceived by some random vehicles in the pre-destined pathway. The hardware equipped with extensive storage, RAM, and intensified computation capabilities which is greater than a Home Personal Computer, takes care of admonishing the algorithm which fabricates the pathway embedded with the concept of optimality, and it is the sole responsibility for pinpointing an alternative gilt-edge path way for a one unique Paramedical
Vehicle in the event of a mishap or a bottleneck in the flow of traffic. For the purpose of simplifying and comprehending the aspect of technicality, a tuple filled with documentations would deem quite fruitful as picturized under the Table numbered by the numeral 1 . The Algorithm destined for outlining a pathway which is optimal in nature is prepared in a consuetude that which equates two entities one wards off the bottleneck caused by the flow of traffic, thereby ensuring the contraction related to the amount involved in traversing from one point to another. Therefore, with a reference to the above dual entities get suggested in this juncture, one refers to the weighted median cadent based on the cost, two points to the inclination of the person behind the steering wheel. A few of the documentations which are inherent in Mathematics get utilized with the Algorithm, in turn represent a sampled value with time, segments on the Pathway, point of termination of a journey, and last the four wheelers, getting represented by mathematical notations like $T,(\mathrm{i}, \mathrm{j}), \mathrm{d}$ and last but not the least V. At this juncture, the length or the distance from one point to the next termination point gets taken into account and gets denoted by a mathematical notation PLVd (check) $c i$, the pathway which gets elected way ahead of the replanning could happen, is given out by the notation $\mathrm{ci}$. The pathway which has undergone a change is singled out by the notation PLVdpij, the newest pathway which is conceived by a collective idea or a plan gets denoted by $p i j$, in the event a four wheeler traverses through the segments of the pathway denoted by $(i, j)$ the path taken by the four wheeler gets correlated alongside the path way of $\mathrm{d}$. The cost associated with the traversal of four wheeler from one point to the next point gets denoted by the notation $(T)$ and is derived by the following expression

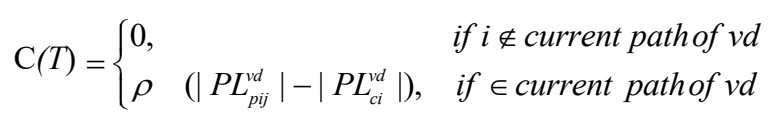

with the reference to the Manuscript numbered [37] the notation utilized to calculate the addition of length in the pathway is pointed out by $\rho$.

Table 1. Summary of the important mathematical notations

\begin{tabular}{c|l}
\hline Symbol & \multicolumn{1}{|c}{ Description } \\
\hline $\mathrm{v}$ & Vehicles \\
$\mathrm{d}$ & Destination \\
$(i, j)$ & Road segment \\
$\mathrm{T}$ & Sample time duration \\
$P L_{p i j}^{v d}$ & Prevailing length of the path \\
$c i$ & The path selected ahead of the re-planning \\
$P i j$ & The changed path \\
$C(T)$ & Vehicle travel cost \\
$\rho$ & A nonnegative accumulative function \\
$C_{i j}(T)$ & The average cost of vehicles on road segment \\
$C_{i}(T)$ & The average cost of vehicles on intersection $i$ \\
$J_{i}$ & The set of adjacent crossing of intersection $i$ \\
$\lambda_{i j}(T)$ & The average inflow rates of road segment \\
$\mu_{i j}(T)$ & The average outflow rates of road segment \\
$\lambda_{i j}(T)$ & The upstream vehicle arrivals respectively on road \\
$\mu_{i j}(T)$ & segment \\
& The upstream vehicle departure rates on road \\
$\Delta$ & segment \\
$Q_{i}(T)$ & Sample time duration \\
$W I$ & The total length of all virtual queue intersection $i$ \\
$W I(T)$ & Weighted intersection \\
\hline
\end{tabular}


$Q_{i}^{d}(T) \quad$ Virtual queue representing the number of shielded vehicles travelling through intersection $i$

$Q_{j}^{d}(T) \quad$ Virtual queue representing the number of shielded vehicles travelling through intersection $j$

$j^{*} \quad$ The huge differential row build-up for intersection $i$ and the other adjacent intersection $j$

$q_{j^{*}}^{d}(T) \quad \begin{aligned} & \text { Re-planning to intersection } j^{*} \text { of a number of } \\ & \text { vehicles }\end{aligned}$

The calculation of the median cost of traversal required for a four wheeler based on a segment on the road denoted by $(i, j)$ and is derived by the following Expression

$$
C_{i j}(T)=\frac{\sum C(T)}{V}
$$

the notation pointing the alphabet $V$ denotes the value on how many four wheelers traverse on the segment of the Road. When deciding up on a junction, acknowledging that there might exist more than one adjoining junctions which turn out to be the applicants to the junction being beseeched, ascertaining the median tariff associated to the vehicles with regard to the junction $i$ is expressed out as follows

$$
C_{i}(T) \frac{\sum_{j \in J_{i}} C_{i j}(T)}{J_{i}}
$$

the notation which is utilized for pin pointing the batch of two neighboring crossing of junctions $i$ gets denoted by $J$ suffix(check)i. Furthermore, in accordance to Manuscript number [38] the diagram christened under the numeral value 4 describes a methodology for blueprinting the flow of a particular traffic. With the reference to the rate of flow of traffic as well as the interval of time denoted by the alphabet ' $\mathrm{T}$ ' which was basketed by the Units placed on the sides of the Roads, the rate associated with the in and outflow of the segment of the pathway are apparently lambda showcased as $i(T)$ and $\mu i j(T)$, and the above is expressed as follows

$$
\lambda_{i j}(T)=\frac{1}{\Delta} \sum_{t=(T-1) \Delta}^{T \mathrm{~A}} \lambda_{i j}(t)
$$

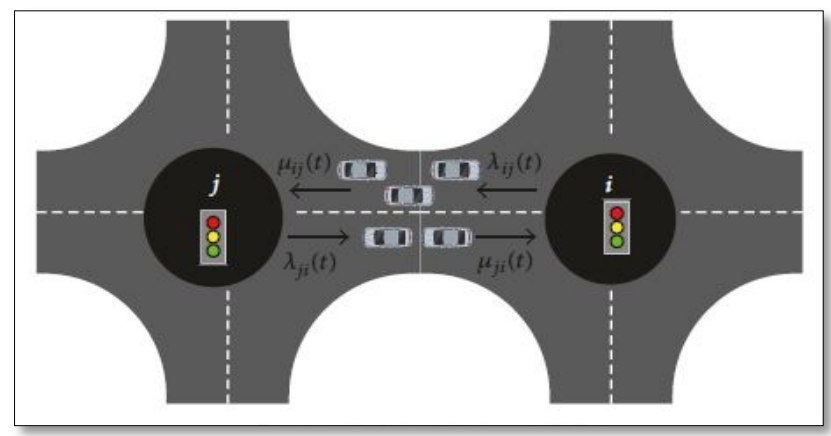

Fig.4. Traffic flow model

$$
\mu_{i j}(T)=\frac{1}{\Delta} \sum_{t=(T-1) \Delta}^{T \Delta} \mu_{i j}(t)
$$

the notations lambda $\lambda$ suffix $i j(t)$ as well as $\mu$ suffix $i j(t)$ connote dual entities, one being the arrival of four wheelers from upstream and two is the rate of departure based on the segment of the pathways $(i, j)$, and the termination point being the same with reference to the time slot denoted by the notation $t$. The sampled duration with respect to time that embeds an array of slots associated with the timing which is acclaimed by a theorem termed as Sampling, that has an intent of diminishing the liability concerned with debt of data which is related to the reckoning of the flow of traffic, given under the Manuscript numbered [39]. It is quite trivial to be aware of the fact that the entire set of variables associated to the segment of the pathway denoted as $(i, j)$ on the counter route which is being labeled as segments on the $\operatorname{Road}(j, i)$ may get outlined as lambda $\lambda j i(T)$ as well as $\mu j i(T)$. For the purpose of determining if a junction is durable as well as reliable, an entity defined as the data on top of a queue being virtual with a specific length is needed and the notation which is used to denote it is given as $Q(T)$. For the purpose of detailing the number of four wheelers left out on the segment of a pathway $(i, j)$ is expressed as follows:

$$
Q_{i j}(T)=\lambda_{i j}(T-1)+\mu_{i j}(T-1)
$$

With the reference to the Expression represented by the numeral 5, it is quite possible to retrieve the complete magnitude of the ' $n$ ' number of queues being virtual in nature which are associated with the junction $i$ with reference to all terminal end points as given below:

$$
Q_{i}(T)=\sum_{j \in J_{i}} Q_{i j}(T)
$$

Catered and if conditions which are deemed suitable prevails for the aspect of stable conditions throughout the ' $n$ ' number of queues which are inherent inside a network, then it is taken hands down, that the flow of traffic with reference to the entire network will be completely durable as well as reliable. Given a circumstance that the Centralized Machine deemed as the Server gets pinged with a red alert from a collectivity of four wheelers via the VANET as well as the units placed on the sides of the Roads, the Algorithm planning the Route embedded with optimality gets evaluated for updation as well as to ascertain the value denoted by $\lambda i(T)$ with the intention of contracting the tariff for traversing as well as bettering the utilization of space. With a concern to the entity of time with regard to the sample denoted by ' $\mathrm{T}$ ' which is shouldered on the Algorithm planning the Route embedded with optimality, it is absolutely attainable for hastening a four wheeler and given its end point denoted by the notation $d$ commencing from one particular junction onwards another. The four wheeler under study gets maneuvered via the revised pathway as and how the Global Positioning System shows. For the purpose of catering the objective of dual entities, one being the contraction of the tariff required by the four wheeler and two the utilization of space, a novel cadent gets conceived and it is defined as a junction based upon the weights, denoted by WI. In the event of letting the notation $\mathrm{WI}(T)$ to illustrate the weight associated with a junction $(i)$ with reference to a sampled time denoted by the notation $T$, which is expressed below as

$$
W I_{i}(T)=\left[Q_{i}^{d}(T)-Q_{j}^{d}(T)\right]-K C_{i}(T)
$$

the dual notations showcased as $Q \operatorname{di}(T)$ as well as $Q d j(T)$ which are being utilized for singling out the queues being virtual, in turn are used in a manner to portray the ' $n$ ' number of four wheelers which are being concealed and passed via 
the junctions onwards the path of the notation $d$ within the sampled time denoted by the alphabet $T$. The Server present in the Centralized Location provisions a constant denoted by the alphabet, gets utilized by each and every unique four wheeler, as mentioned under the Manuscript enumerated under [40]. With reference to the inception, the four wheelers traversing via a particular junction possessing a humongous load gets reassessed. Ultimately, the four wheelers that have the terminal point denoted by the alphabet $d$, positioned on $i$, ought to get fast forwarded to a particular queue denoted by the notation $Q d j *(T)$ with a junction denoted by $j *$, in turn delineates the humongous row which is developed on account of the junction denoted by $i$ as well as an alternative junction given as $j$ and last but not the least the terminal point $d$ expressed as follows:

$$
j^{*}=Q_{i}^{d}(T)-Q_{j}^{d}(T)
$$

With reference to the hike in the terse length of the pathway, there occurs a remodeling based on the junction denoted by $j *$ which is associated with ' $n$ ' number of four wheelers where the terminal point gets denoted by $d$, by means of the given expression

$$
q_{j^{*}}^{d}(T)=Q_{i}^{d}(T)-Q_{j^{*}}^{d}(T)
$$

Later, the queues which are present in the left over junctions gets refreshed by means of the following:

$$
\begin{aligned}
& Q_{i}^{d}(T)=Q_{i}^{d}(T)-q_{j^{*}}^{d}(T) \\
& Q_{j^{*}}^{d}(T)=Q_{j^{*}}^{d}(T)+q_{j^{*}}^{d}(T)
\end{aligned}
$$

A procedure which is identical to the existing one goes on and on, until all the junctions which are chained to one another have undergone the procedure. The Algorithm pointed by the numeral 1 displays the specific aspect of ORPA.

\section{Performance Evaluation}

In order to perform a trial and error scrutiny, a grid segment based out of Manhattan got chosen. For the purpose of fabricating the sequence of events as well as to pull out the pictures or bit map images which are associated with the intersection. Dual Applications get utilized for the above requirement which are termed as Maps from Google as well as MatLab. Virtual Reality replica blows out a segment related to providing assistance to mobility, that has the efficiency to configure the four wheelers to step on the gas, configure the flow of the vehicle, reduce the rate of acceleration, and ' $n$ ' number of real time events can get implemented in a sand box environment. With reference to this section of the scrutiny, an identical mobile segment related to the Four wheeler blueprint mentioned by the author in [4] gets utilized. Utilizing the median rate of acceleration data, which was taken from the units placed at the side of the roads, the digitized chart of the Road gets bifurcated into dual zones of acceleration. One being congested and the other being unhampered with congestion. Given a section of the pathway, if there exists a lower rate of acceleration among the four wheelers; it is undeniably congested, else it is free from movement bottleneck. Nonetheless, the methodology of recording the data associated with the median rate of acceleration of perpetual four wheelers might possess some wrong entries, on account of the previously recorded data. The aftermath of the above data possess the traits of information from the past and not of the data availed at hand in the present. For the purpose of alleviating the short coming, segregations are conceived based on the $2 \times 2.5$ minute time window, coupled with the evaluation of cumulative median rate of acceleration, with the piece of data derived from that particular time window. The other remonstrance is the formation of accumulated data, which results from the updates which are related to the acceleration being performed at regular intervals between the units placed at the side of the roads and the Centralized Server. In order to undertake and handle this remonstrance the rate of acceleration gets bifurcated by means of zones relative to the time, provided the units along the sides of the Roads churn in the data referring only to the median rate of acceleration and only if there exists a steady rise and decrease with reference to one zone to the other in terms of the time. Dependent on the units placed on the sides of the roads, the Centralized Server present in the equation provisions the information regarding the updation of the zones with respect to Time on par with the bottleneck relevant to the section of the Road. A compare and contrastive table based upon the assessment of Quadra Algorithms, which are going to get utilized within the configuration of the Paramedical Vehicle by means of utilizing the dual entities; median rate of acceleration as well as the time frame required by the Medical Team to arrive gets conceived. The Algorithm termed as Dijkstra spans out the traits for the purpose of blueprinting the pathway sans any sort of modifications. In this scenario, each and every unique road section associated with the acceleration of the four wheelers implants a unique median numeral for all the sections which are available for the four wheelers to traverse through. In the event of utilizing the algorithm termed Djikstra, the entity defined as grid lock caused by the stagnant four wheelers doesn't get considered, the rate of acceleration can never undergo any sort of a modification. There exists certain parallelism or degree of similarity in comparison between $\mathrm{V}^{*}$ as well as Dijkstra Algorithm. Nonetheless, with the reference to the $\mathrm{V}^{*}$ Algorithm a unique function depicted by $\mathrm{L}(\mathrm{n})$ gets provisioned. The above function is utilized to connote an angle which exists between dual entities, one being the neighboring node and two being the unwavering line pointing the path onwards the node depicted as the end point. With the reference to the Algorithm defined as VBA\#, the entity defined as acceleration which is prevalent on top of a section for a pathway, has the ability to get configured in a dynamic way, added to the above an entity defined as a manner based on distribution can get utilized to perform some functionalities. A compare and contrast activity gets performed, among the entities defined as $\mathrm{V}^{*}$, VBA\#, ORPAA, and last but not the least the Dijkstra Algorithm. The Virtual implementation aftermath is depicted under the Table pointed by the Numerical value 2.The figure represented by the numeral 5 denotes the value of time utilized by the Quadra Algorithms for traversing from the source to the destination with respect to dual entities (i) Heavy bottleneck conceived by the flow of traffic (ii) Full fledged free flow of Vehicles. The algorithm termed ORPAA achieves a reduced value in time for traversing from a source to the destination than when compared to its trio counterparts. With the reference to the (ii) Free flow of Vehicles the traversal value in terms of the measurement of seconds turned out to be 1300, 2300, 2100 and last but not the least numeral turned out to be 1900 . 


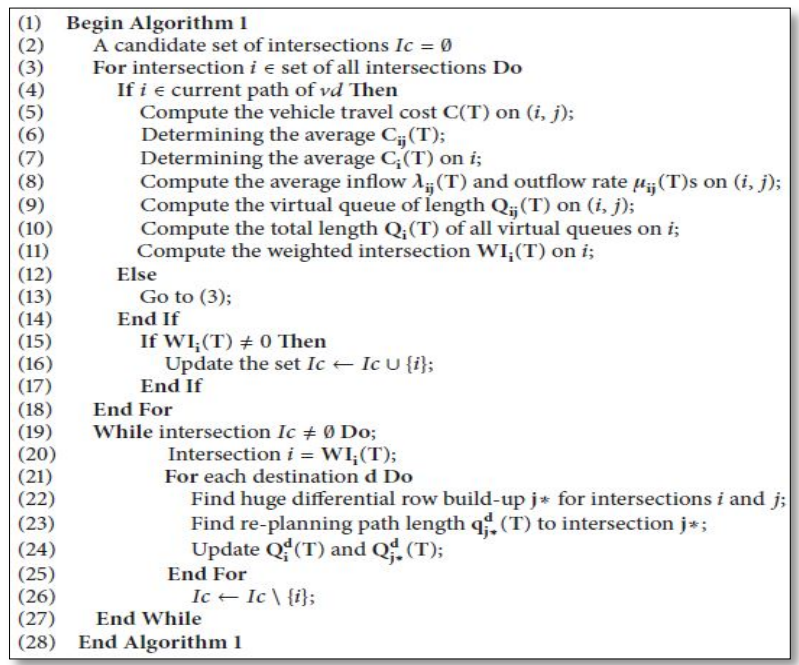

Table 2. Simulation parameters

\begin{tabular}{l|c}
\hline \multicolumn{1}{c|}{ Parameters } & Value \\
\hline Simulation area & $5 \times 5 \mathrm{~km}^{2}$ \\
Number of lanes & 2 bidirectional \\
Intersections & 24 \\
Segments & 38 \\
Speed & $(40-80) \mathrm{km} / \mathrm{hr}$. \\
Average vehicles density & High density \\
& $40-50 \mathrm{veh} / \mathrm{lane} / \mathrm{km} \&$ low \\
Communication radius & Density $6-8 \mathrm{veh} / \mathrm{lane} / \mathrm{km}$ \\
MAC protocol & $300 \mathrm{~m}$ \\
Total simulation time & IEEE $802.11 \mathrm{p}$ \\
\hline
\end{tabular}

The diagram depicted under the numeral 5 brings out the obvious fact that the traversal time raises above the expected threshold value with reference to (i) and simmers down with respect to (ii). The Diagram depicted under the numeral number 6 show cases the average rate of acceleration of a Paramedical Vehicle from the Health Care Building onwards to the scene of the Human Casualty with reference to dual scenarios.

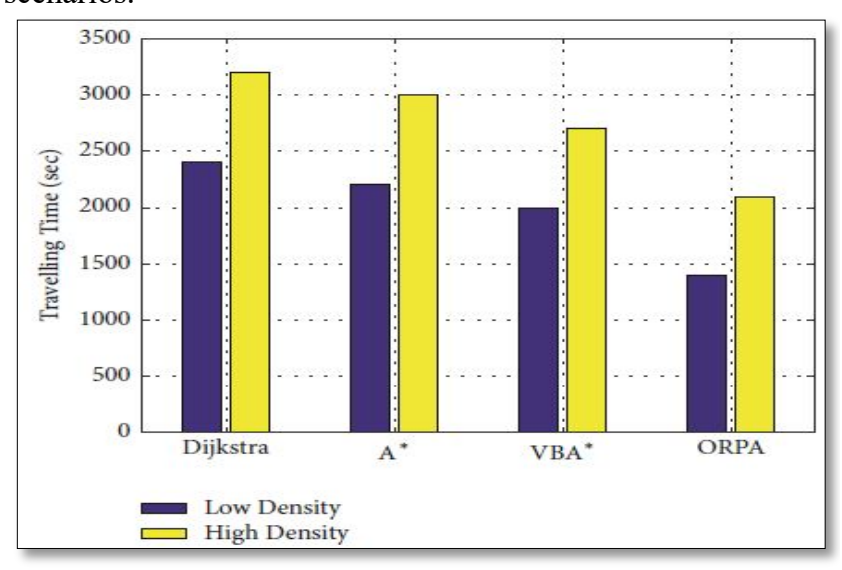

Fig. 5. Travelling time in low-and high-density

The Algorithm termed as ORPAA takes the limelight in terms of several entities like the average rate of acceleration, the percentile comparison between the other Algorithms, the time recorded by the Healthcare Unit in reaching the scene of incident and so on.

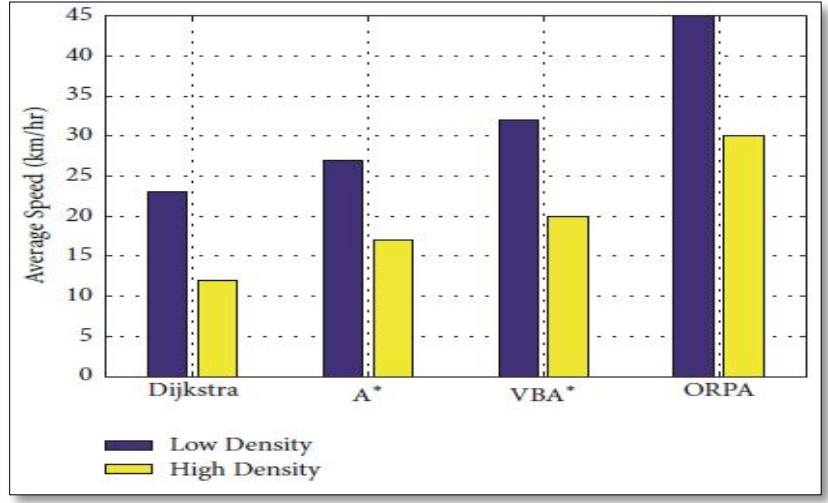

Fig. 6. Average ambulance speed in low - and high-density

The diagram depicted under the numeral 6 brings out the obvious fact that the average rate of acceleration of a Paramedical Vehicle with respect to roman numeral ii is way more than when compared with the roman numeral $i$.

\section{Conclusion}

With reference to this Manuscript, we have gone ahead and conceived an orderliness which is commonly utilized in governing the mishaps that arise on account of the four wheelers, which in turn gives an opportunity for several vehicles to avert the neighborhood or regions which are deem to be crowded or bottlenecked residing with respect to an ITS. At the outset, we had chartered an orderliness that governs over the mishaps caused by the four wheelers which in turn utilizes a particular system made up of Cellular Technology which is associated with the transportation utilized by people as well as the entity defined as VANET for conceiving a competent on the fly exchange of digital data in between several vehicles like the Paramedical Six Wheelers, Health Care Units, Units placed along the side of the Roads, as well as the Server placed in a Centralized Location. On the other hand consequently we are provisioning an on the fly contrivance for devising pathways with the intent of bettering the comprehensive space utilization, whilst compacting the tariff required for traversal from one point to the other, via the caliber of the four wheelers to avert the block of pathways which is crowded by ' $n$ ' number of vehicles. Last but not the least, it is highlighted that the contrivance which takes care of devising the path way, being provisioned here would diminish the interval of time utilized by the Paramedical Six wheelers, from the time when a data regarding a mishap gets shot to the Health Care Centre, to the time when the Paramedical Vehicle reaching the exact scene of the incident, can get completely achieved by averting the blocks of pathways which are gridlocked, which in turn catapults the opportunity of preserving the lives of individuals caught in mishaps, from 60 to 98 percentile.

This is an Open Access article distributed under the terms of the Creative Commons Attribution License

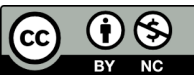




\section{References}

[1] M. Y. I. Idris, A. M. A. Abu Znaid, A. W. A. Wahab, L. K. Qabajeh, and O. A. Mahdi, "Low communication cost (LCC) scheme for localizingmobilewireless sensor networks," Wireless Networks, vol. 23, no. 3, pp. 737-747, 2017.

[2]. A. S. Sadiq, S. Khan, K. Z. Ghafoor, M. Guizani, and S. Mirjalili, "Transmission power adaption scheme for improving IoV awareness exploiting: evaluation weighted matrix based on piggybacked information," ComputerNetworks, vol. 137, pp. 147159, 2018.

[2] G. Egilmez and D. McAvoy, "Benchmarking road safety of U.S. states: A DEA-based Malmquist productivity index approach," Accident Analysis \& Prevention, vol. 53, pp. 55-64, 2013.

[3] H. Hartenstein and K. Laberteaux, VANET: Vehicular Applications and Inter-Networking Technologies, vol. 1, John Wiley \&Sons, 2009.

[4] Y. R. B. Al-Mayouf, N. F. Abdullah, M. Ismail,A. W.A. Wahab, and O. A. Mahdi, "Efficient routing algorithm for VANETs based on distance factor," in Proceedings of the 2016 InternationalConference on Advances in Electrical, Electronic and SystemsEngineering, ICAEES 2016, pp. 567-571,mys, November 2016.

[5] Y. Sun, R. Lu, X. Lin, X. Shen, and J. Su, "An efficient pseudonymous authentication scheme with strong privacy preservationfor vehicular communications," IEEE Transactions on VehicularTechnology, vol. 59, no. 7, pp. 3589-3603, 2010.

[6] Y. R. B. Al-Mayouf, M. Ismail, N. F. Abdullah, S. M. Al- Qaraawi, and O. A. Mahdi, "Survey on VANET technologies and simulation models," ARPN Journal of Engineering andApplied Sciences, vol. 11, no. 15, pp. 9414-9427, 2016.

[7] M.Wang, H. Liang, R. Zhang, R. Deng, and X. Shen, "Mobility aware coordinated charging for electric vehicles in VANET enhanced smart grid," IEEE Journal on Selected Areas in Communications, vol. 32, no. 7, pp. 1344-1360, 2014.

[8] Leontiadis, G. Marfia, D. Mack, G. Pau, C. Mascolo, and M. Gerla, "On the effectiveness of an opportunistic traffic management system for vehicular networks," IEEE Transactionson Intelligent Transportation Systems, vol. 12, no. 4, pp. 1537-1548, 2011.

[9] O. A. Mahdi, A. W. Abdul Wahab, M. Y. I. Idris et al., "A comparison study on node clustering techniques used in target tracking WSNs for efficient data aggregation," WirelessCommunications and Mobile Computing, vol. 16, no. 16, pp. 2663-2676, 2016.

A. Skabardonis and N. Geroliminis, "Real-time monitoring and control on signalized arterials," Journal of IntelligentTransportationSystems: Technology, Planning, and Operations, vol. 12, no.2, pp. 64-74, 2008.

[10]N. M. Drawil and O. Basir, "Intervehicle-communication assisted localization," IEEE Transactions on Intelligent TransportationSystems, vol. 11, no. 3, pp. 678-691, 2010.

[11]O. A. Mahdi, A.W. AbdulWahab, M. Y. I. Idris, A. Abu Znaid, Y. R. B. Al-Mayouf, and S. Khan, "WDARS: a weighted data aggregation routing strategy with minimum link cost in eventdrivenWSNs," Journal of Sensors, vol. 2016, Article ID 3428730, 12 pages, 2016.

A. M. A. Abu Znaid, M. Y. I. Idris, A. W. Abdul Wahab, L. KhamisQabajeh, and O. Adil Mahdi, "Sequential Monte Carlo Localization Methods in Mobile Wireless Sensor Networks: A Review," Journal of Sensors, vol. 2017, Article ID 1430145, 2017.

[12]H. Khosroshahi, P. Keshavarzi, Z. D. Koozehkanani, and J. Sobhi, "Acquiring real time traffic information using VANET Journal of Advanced Transportation 11 and dynamic route guidance," in

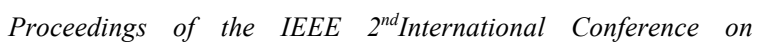
Computing, Control and IndustrialEngineering, CCIE 2011, pp. 913, August 2011.

[13] O. A. Mahdi, A. W. Abdul Wahab, M. Y. I. Idris, A. Abu Znaid, S. Khan, and Y. R. B. Al-Mayouf, "ESAM: endocrine inspired sensor activation mechanism for multi-target tracking inWSNs," in Proceedings of the 4th International Conference onWireless and Optical Communications, vol. 9902 of Proceedingsof SPIE, International Society forOptics and Photonics, Beijing, China, June 2016.

[14] M. Abboud, L. Jaoude, and Z. Kerbage, "Real time GPS navigation system," in Proceedings of the 3rd FEA Student Conference, Department of Electrical and Computer Engineering, American University of Beirut, Citeseer, 2004.

[15] Y. Chung, "Development of an accident duration prediction model on the Korean Freeway Systems," Accident Analysis \&Prevention, vol. 42, no. 1, pp. 282-289, 2010.

[16] Y. Zhao, K. Triantis, D. Teodorovi'c, and P. Edara, "A travel demand management strategy: The downtown space reservation system,'European Journal of Operational Research, vol. 205, no. 3, pp. 584-594, 2010.

[17] C. Herrera, D. B. Work, R. Herring, X. Ban, Q. Jacobson, and A. M. Bayen, "Evaluation of traffic data obtained via GPSenabledmobile phones: the Mobile Century field experiment," Transportation Research, Part C: Emerging Technologies, vol. 18, no. 4, pp. 568583, 2010.

[18]R. Herring et al., "Using mobile phones to forecast arterial traffic through statistical learning," in Proceedings of the $89^{\text {th }}$ Transportation Research Board Annual Meeting, 2010.

[19] Jariyasunant et al., Mobile transit trip planning with real-time data, 2011, Mobile transit trip planning with real-time data.

[20]Hoh, M. Gruteser, R. Herring et al., "Virtual trip lines for distributed privacy-preserving traffic monitoring," in Proceedingsof the 6th international conference on Mobile systems,applications, and services, p. 15, Breckenridge, CO, USA, June 2008.

[21] H. T. Cheng, H. Shan, and W. Zhuang, "Infotainment and road safety service support in vehicular networking: from a communication perspective," Mechanical Systems and SignalProcessing, vol. 25, no. 6, pp. 2020-2038, 2011.

[22]J. Zhao and G. Cao, "VADD: vehicle-assisted data delivery in vehicular Ad hoc networks," IEEE Transactions on VehicularTechnology, vol. 57, no. 3, pp. 1910-1922, 2008.

[23] T. H. Luan, X. Ling, and X. Shen, "Provisioning QoS controlled media access in vehicular to infrastructure communications," $A d$ Hoc Networks, vol. 100, no. 2, pp. 231-242, 2012.

[24]Xu, F. Zhao, J. Guan, H. Zhang, and G.-M.Muntean, "QoEdrivenuser-centric vod services in urban multihomed P2Pbased vehicular networks," IEEE Transactions on VehicularTechnology, vol. 62, no. 5, pp. 2273-2289, 2013.

[25]Zhou, Y. Zhang, K. Song, W. Jing, and A. V. Vasilakos, "Distributed media services in P2P-based vehicular networks," IEEE Transactions on Vehicular Technology, vol. 60, no. 2, pp.692-703, 2011.

[26] P. Chen, Y. Guo, andW. Chen, "Fuel-Saving Navigation System in VANETs," in Proceedings of the 2010 IEEE Vehicular TechnologyConference (VTC 2010-Fall), pp. 1-5, Ottawa, ON, Canada, September 2010.

[27] M.Kimura, Y. Taoda, Y. Kakuda, S. Inoue, andT. Dohi, "Anovelmethod based on VANET for alleviating traffic congestion in urban transportations," in Proceedings of the 2013 IEEE Eleventh. 